\title{
(6) OPEN ACCESS \\ Improving outcomes in patients with Acute Kidney Injury: the impact of hospital based automated AKI alerts
}

\author{
M Prendecki, ${ }_{1}^{1}$ E Blacker, ${ }^{2}$ O Sadeghi-Alavijeh, ${ }^{1}$ R Edwards, ${ }^{3}$ H Montgomery, ${ }^{2}$ \\ $S$ Gillis, ${ }^{2}$ M Harber ${ }^{4}$
}

'Department of Nephrology, Whittington Hospital, London, UK

${ }^{2}$ Department of Intensive Care Whittington Hospital, London, UK

${ }^{3}$ Department of Biochemistry, Whittington Hospital, London, UK

${ }^{4} \mathrm{UCL}$ Centre for Nephrology, Royal Free Hospital, London,

Correspondence to Dr M Prendecki, Department of Nephrology, Whittington Hospital, London N19 5NF, UK; maria.prendecki@nhs.net

Received 22 April 2015 Revised 24 September 2015 Accepted 25 September 2015 Published Online First 28 October 2015

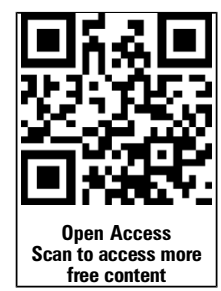

CrossMark

To cite: Prendecki M, Blacker E, Sadeghi-

Alavijeh 0, et al. Postgrad Med J 2016:92:9-13.

\section{ABSTRACT}

Background Acute kidney injury (AKI) is a significant cause of morbidity and mortality. Early identification may improve the outcome and in 2012 our hospital introduced an automated AKI alert system for early detection and management of AKI.

Objectives Using an automated AKI alert system we analysed whether early review and intervention by the Critical Care and Outreach (CCOT) team improved patient outcomes in AKI and whether serum bicarbonate was useful in predicting outcomes in patients with AKI. Methods In a retrospective analysis we identified patients who triggered an AKI alert from 20 April 2012 to 20 September 2013 and collected data on mortality, length of stay, need for intensive care admission and renal replacement therapy (RRT).

Results 994 AKI alerts were generated and analysed. Patients with bicarbonate outside the normal range had significantly higher mortality. Bicarbonate $<22 \mathrm{mmol} / \mathrm{L}$ was associated with a mortality of $25.7 \%$ (49/191) compared with $16.9 \%$ (39/231) when $22-29 \mathrm{mmol} / \mathrm{L}$ $\left(p=0.047, \chi^{2}\right)$. Those patients reviewed $\geq 1$ day after AKI alert by CCOT compared with those seen on the day of the alert had a 2.4 times increase in mortality and were 7 times more likely to require RRT acutely.

Conclusions Electronically identified AKI alerts identify patients at high risk of morbidity and mortality. In this group AKI alerts preceded CCOT review by a mean of 2 days. This represents a window for supportive interventions, which may explain improved outcomes in those reviewed earlier. The addition of serum bicarbonate offers a further method of risk stratifying patients at greater risk of death.

\section{INTRODUCTION}

Acute kidney injury (AKI) is common in hospital with recent multicentre studies reporting rates of up to $7 \%$ in the USA. ${ }^{1-3}$ It is also increasingly recognised as a very significant cause of morbidity and mortality in hospital inpatients. Multiple studies have shown inhospital and 90 day mortality of around $20-25 \%$, and $50 \%$ for those requiring acute renal replacement therapy (RRT). ${ }^{45}$ It is also clear that AKI is associated with development of chronic kidney disease (CKD) or worsening of existing CKD even in those who recover renal function after an episode of AKI. ${ }^{67}$ Rates of end-stage renal disease and long-term mortality are also higher. ${ }^{89}$

AKI is estimated to cost the National Health Service (NHS) $£ 620$ million/year ${ }^{10}$ and yet despite the high mortality and impact of AKI, early identification of at-risk patients has been poor. A
National Confidential Enquiry report in 2009 highlighted several deficiencies in the care of patients with AKI including delayed identification and delayed referral for specialist input. ${ }^{10}$ Given the paucity of effective medical treatments for established AKI, early detection and supportive interventions are likely to be critical; this has promoted the implementation of 'AKI alerts' to aid early detection and management. ${ }^{11}{ }^{12}$ A national patient safety alert issued in June 2014 has recommended that all NHS hospitals in England, providing pathology services, implement an algorithm for AKI alerting by March 2015 (see box 1). ${ }^{13}$

From April 2012 our hospital has operated an automatic alerting system whereby every creatinine processed is compared with the previous (if available within the preceding 90 days). If the current value is $\geq 150 \%$ the previous, an automated message is generated and attached to the report. Since January 2013 an additional test for venous bicarbonate is automatically added to any biochemistry request triggering an AKI alert. Our study had two objectives; first, was the additional, (unrequested) result for venous bicarbonate useful in determining which patients were more likely to require Intensive treatment unit (ITU) admission, acute RRT or have increased mortality? Our second objective analysed a subgroup of patients who had AKI but were also reviewed by Critical Care and Outreach (CCOT). We aimed to identify if in these patients early intervention by CCOT was associated with improved patient outcome, mortality or reduced need for ITU admission and acute RRT compared with later assessment.

\section{METHODS}

Patients

A database of all patients who triggered an AKI alert between 20 April 2012 and 20 September 2013 was generated by the biochemistry laboratory. Alerts generated for patients in the outpatient department, general practice or emergency department were excluded if the patients were not admitted to hospital within $24 \mathrm{~h}$. Patients in whom the AKI alert was artefactual were excluded, this included patients who were established on RRT (either acute or chronic), patients with a single spuriously low or high creatinine, postpartum women and patients with icteric or lipaemic samples.

\section{Subgroup of patients referred to CCOT}

By cross-referencing the AKI database with the clinical database for CCOT referrals we identified a 


\section{Box 1 National Patient Safety Alert June 2014}

- Issued to all NHS England Hospitals

- A national algorithm standardising the definition of AKI has been agreed

- Algorithm should be integrated into a laboratory information management system

- Priority is adoption of an e-alert system which notifies clinicians when a patient has AKI

subgroup of patients who triggered an AKI alert but were also seen by CCOT on the basis of a physiological deterioration. AKI alerts were not part of CCOT referral criteria at this time. Patients who were seen by CCOT as part of routine step-down from ITU care were excluded. Data were collected retrospectively on stage of AKI, length of stay, trigger for and timing of CCOT review, ITU admission and mortality.

\section{Clinical data}

Data were collected from patients' electronic records on age, gender, stage of AKI, venous bicarbonate, mortality, admission to ITU and acute RRT. Stage of AKI was defined using KDIGO biochemical staging criteria for severity of AKI (stage 1 corresponds to serum creatinine 1.5-1.9 times baseline, stage 2, 2.0-2.9 times baseline and stage 3, >3.0 times baseline). Progression of AKI from stage 1 to stage 2 or stage 3 was identified by looking at serial creatinine results over subsequent days for individual patients after the AKI alert was generated. Patients were identified who had AKI stage 1 but then had a subsequent creatinine which when compared with the initial baseline would correspond to AKI stage 2 or stage 3 .

\section{Data analysis}

Stage of AKI, mortality and proportion of patients needing ITU admission and acute RRT were calculated and compared between patients with normal bicarbonate $(22-29 \mathrm{mmol} / \mathrm{L})$, those with low bicarbonate $(<22 \mathrm{mmol} / \mathrm{L})$ and high bicarbonate (>29 mmol/L).

Patients reviewed by CCOT were divided into two groups, those seen prior to or on the day of the AKI alert and those seen after the AKI alert. Stage of AKI, mortality, need for ITU admission, acute RRT and length of hospital stay were compared between the two groups.

\section{Statistical analysis}

Data are reported as mean values with range unless otherwise stated. Student's $t$ test was used for continuous variables and $\chi^{2}$ test for the difference in proportions between two groups. For $\chi^{2}$ tests where the contingency table is larger than $2 \times 2$, Bonferroni correction is used for post hoc analysis. Results are reported as statistically significant when $\mathrm{p}<0.05$.

\section{RESULTS \\ Alerts}

Over the 16-month period of analysis, there were 1421 AKI alerts generated; 272 of these were in outpatients or patients seen in the emergency department but not admitted, 79 were in pregnant or postpartum women and 76 alerts were excluded as not a true AKI. Of the 76 excluded, 27 were because the creatinine result was the patient's actual baseline or there was clearly an erroneous result (in some cases this was documented as a drip arm or diluted sample), 8 alerts were in existing haemodialysis patients, 35 patients were receiving acute haemofiltration in the ITU and in 3 cases the ratio calculated manually was $<1.5$.

\section{Patients}

Nine hundred and ninety-four AKI alerts were included; this represented 940 separate inpatient episodes and 831 different patients. The mean age was 72.27 years (18.3-101.9 years), 437 $(52.6 \%)$ were female and $394(47.4 \%)$ male. Six hundred and thirty (63.3\%) of the alerts were for AKI stage 1, 265 (26.7\%) AKI stage 2 and 99 (10.0\%) AKI stage 3. Of the patients who had multiple episodes of AKI on a single inpatient episode 1 each had four or five AKI alerts, 2 had three AKI alerts and 41 patients had two alerts. Eighty-one patients (9.7\%) had multiple admissions with AKI, of these 1 patient each was admitted five or six times, 2 were admitted four times, 14 on three occasions and 65 were admitted twice.

\section{Time to AKI alert after admission}

Median time to AKI alert after admission was 0 days (0-227 days), 561 patients had an AKI alert at the time of admission and a further 123 patients had an AKI alert within the next $48 \mathrm{~h}$.

\section{Morbidity and mortality}

There were 190 admissions to ITU and 46 patients required acute RRT. Mortality was calculated on the basis of death in hospital and as an outcome of each inpatient episode; overall mortality was $24.9 \%$ (207 patients), in those admitted to ITU mortality was $40.5 \%$ (77 patients) and in patients requiring RRT acutely was $47.8 \%$ (22 patients). Mortality by stage of AKI is shown in figure 1 .

\section{Progression of AKI}

Of the alerts for AKI stage 1, 10.3\% (65) progressed to a higher stage of AKI, 47 of these progressed to AKI stage 2, 9 progressed directly to stage 3 at the next result recorded and 9 progressed to stage 2 and then onto stage 3 . The initial progression in AKI occurred a mean of 2.88 days after the initial AKI alert was generated (0-12 days). The mortality in this group with progressive AKI while an inpatient was $35.4 \%$ overall and mortality in those who progressed from AKI stage 1 to stage 3 was

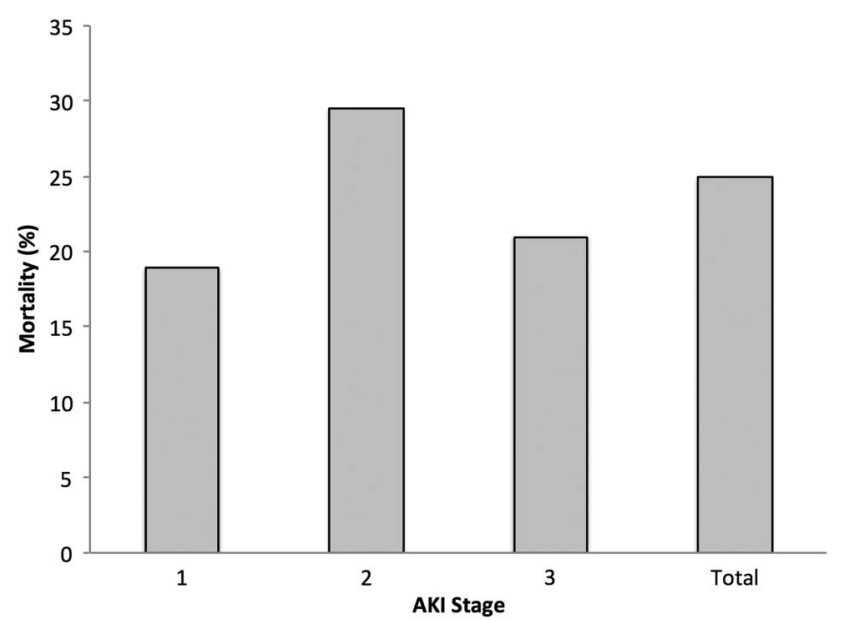

Figure 1 Inpatient mortality by stage of AKI alert. AKI, acute kidney injury. 
significantly higher than those whose AKI was detected initially at stage $3-66.7 \%$ vs $23.9 \%(\mathrm{p}=0.001)$.

\section{Using serum bicarbonate to predict patient outcomes}

Five hundred and twenty-five AKI alerts had a bicarbonate assay added, in 16 cases the sample was not processed due to insufficient serum remaining or the sample being too old giving 509 AKI alerts with results for bicarbonate. For the purpose of analysis of outcomes, where patients had more than one AKI alert for a single admission the bicarbonate and AKI stage for the last recorded episode of AKI was used. One hundred and ninety-one patients had a bicarbonate $<22 \mathrm{mmol} / \mathrm{L}, 23122-29 \mathrm{mmol} / \mathrm{L}$ and $58>29 \mathrm{mmol} / \mathrm{L}$. A higher proportion of patients with low bicarbonate had an AKI stage 3 (11.5\% (22 patients) of patients with bicarbonate $<22 \mathrm{mmol} / \mathrm{L}$ compared with $7.4 \% \quad(17$ patients) of patients with bicarbonate $22-29 \mathrm{mmol} / \mathrm{L}$ ) although this was not significant.

\section{Serum bicarbonate and patient outcome}

Mortality was higher when bicarbonate is outside of the normal range; in those with a bicarbonate $<22 \mathrm{mmol} / \mathrm{L}$ mortality was $25.7 \%$ (49 patients) compared with 16.9\% (39 patients) when bicarbonate was $22-29 \mathrm{mmol} / \mathrm{L}$ and $27.5 \%$ (16 patients) when $>29 \mathrm{mmol} / \mathrm{L}\left(\mathrm{p}=0.047, \chi^{2}\right)$ (figures 2 and 3 ). This was most pronounced in patients with AKI stage 1; mortality 24.3\% (27 patients) when bicarbonate $<22 \mathrm{mmol} / \mathrm{L}, 12.0 \%$ (19 patients) when $22-29 \mathrm{mmol} / \mathrm{L}$ and $25.6 \%$ (10 patients) when $>29 \mathrm{mmol} /$ $\mathrm{L}\left(\mathrm{p}=0.015, \chi^{2}\right)$. Significantly more patients with an abnormal bicarbonate required ITU admission; 25.7\% (49 patients) when $<22 \mathrm{mmol} / \mathrm{L}, 14.7 \%$ (34 patients) when $22-29 \mathrm{mmol} / \mathrm{L}$ and $24.1 \%$ (14 patients) when $>29 \mathrm{mmol} / \mathrm{L}\left(\mathrm{p}=0.015, \chi^{2}\right)$. The need for acute RRT was greatest in those with low bicarbonate compared with the two other groups, $7.3 \%$ vs $2.2 \%$ vs $1.7 \%$ $\left(p=0.019, \chi^{2}\right)$ (figure 3).

\section{Outcome of patients with AKI also referred to CCOT}

Over the same time period 97 patients with an AKI alert were referred to CCOT on the basis of physiological deterioration (such as tachycardia, oliguria, hypotension or hypoxia) or because medical or nursing staff had concerns regarding their condition. The mean age of this group was 71.67 years (28-96 years), $50.5 \%$ patients were female and mean length of stay was 22.4 days (2-163 days). Compared with the whole cohort of patients with AKI there were slightly more patients with an AKI stage 3 and less with an AKI stage 1 (not significant), 57 cases

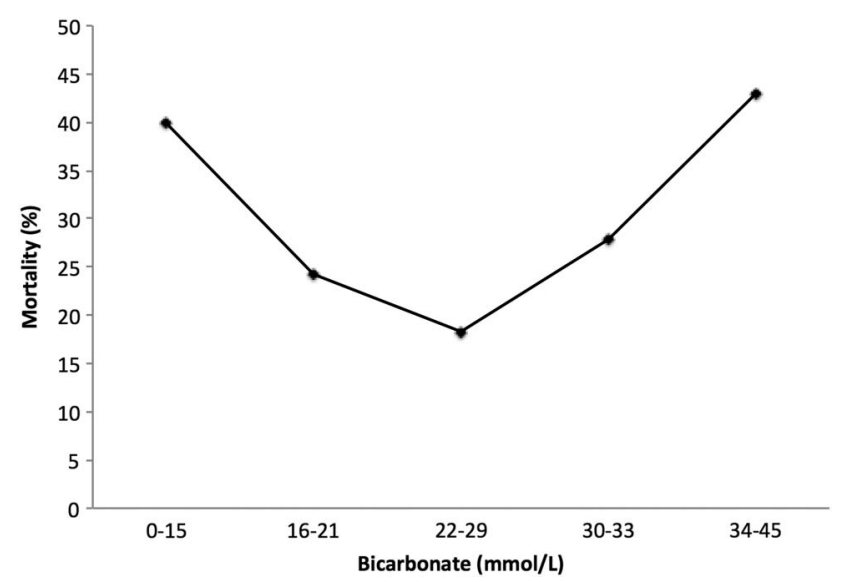

Figure 2 Inpatient mortality stratified by bicarbonate level.
(58.8\%) of AKI stage 1 and 20 cases (20.6\%) each of AKI stage 2 and AKI stage 3. Eighteen (18.6\%) patients were seen postoperatively and $47(48.5 \%)$ had infection or sepsis implicated in the aetiology of their deterioration. Forty-seven patients (48.5\%) reviewed by CCOT required admission to ITU and 13 (13.4\%) acute RRT. The overall inpatient mortality of patients seen by CCOT who also had AKI was $37.1 \%$; this was $33.3 \%$ in AKI stage 1, 45.0\% in AKI stage 2 and $40.0 \%$ in AKI stage 3.

\section{Timing of CCOT review and outcome}

As referral to CCOT was on the basis of physiological decline and not AKI there was often a significant window of time between AKI alert and CCOT review; mean +1.93 days $(-10$ to +33 days). To analyse if this delay to CCOT review altered patient outcomes the patients were stratified into those who were reviewed by CCOT prior to or on the day of AKI alert and those seen on subsequent days (table 1).

Patients seen prior to or on the day of AKI alert had significantly lower mortality, $19.4 \%$ vs $47.5 \%$ in those seen from the day after AKI alert $\left(p=0.0085, \chi^{2}\right)$, and mortality increased with increasing length of time to CCOT review (figure 4 ). This difference was seen in those with AKI stage 1 (13.6\% vs $45.7 \%$, $\left.\mathrm{p}=0.027, \chi^{2}\right)$ and in patients with AKI stage 2 and stage 3 , mortality $28.6 \%$ in those seen prior to or on the day of AKI alert and $50 \%$ in those seen on subsequent days, a large difference although this was not significant due to smaller numbers in this group. Significantly more patients required acute RRT in the group seen later, $19.7 \%$ vs $2.8 \%\left(p=0.04, \chi^{2}\right)$ and this difference was seen in patients with AKI stage 1 and AKI stage 2 and stage 3 (figure 5). In patients who left hospital alive, length of stay was shorter in those who were seen up to and including the day of the AKI alert, 19.3 days vs 24.7 days. In those with AKI stage 2 or stage 3 the difference in length of stay appeared even more pronounced, 15.9 days vs 30.0 days, although these results were not significant as due to high mortality the numbers in each group were small.

\section{DISCUSSION}

In keeping with other published work our study shows that AKI is common and associated with a very high mortality: $24.9 \%$ in the whole cohort rising to $47.8 \%$ in patients who required RRT

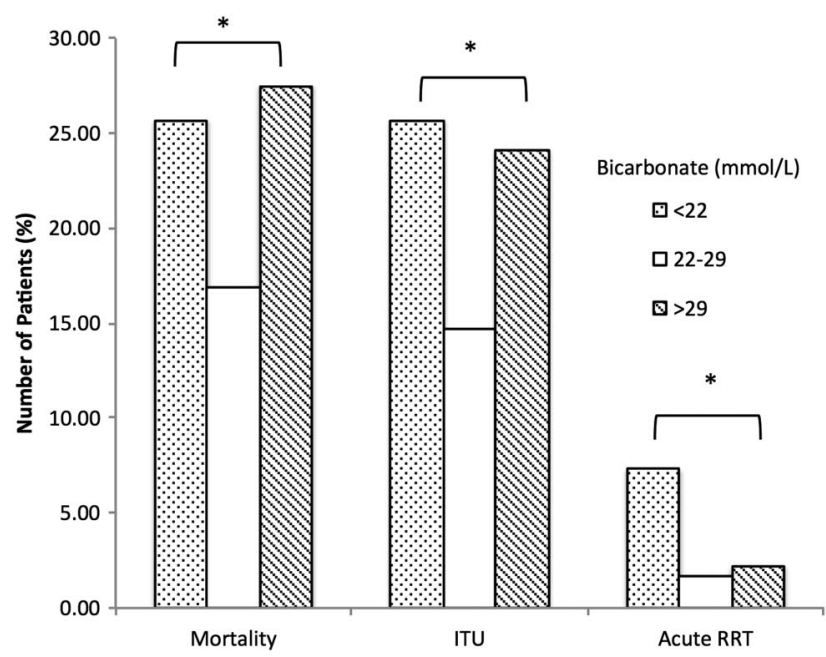

Figure 3 Mortality, need for ITU admission and acute renal replacement therapy (RRT) in all patients compared by bicarbonate level. ${ }^{*} p<0.05$. 
Table 1 Patient demographics when patients are stratified by timing of CCOT review

\begin{tabular}{lll}
\hline & $\begin{array}{l}\text { Seen up to or on day of } \\
\text { AKI alert }\end{array}$ & $\begin{array}{l}\text { Seen after day of } \\
\text { AKI alert }\end{array}$ \\
\hline $\mathrm{N}$ & 36 & 61 \\
Age (years) & $71.81(28.24-96.27)$ & $71.59(30.59-96.80)$ \\
Female/Male & $18 / 18$ & $31 / 30$ \\
AKI stage 1 & $22(61.1 \%)$ & $35(57.3 \%)$ \\
AKI stage 2 & $8(22.2 \%)$ & $12(19.7 \%)$ \\
AKI stage 3 & $6(16.7 \%)$ & $14(22.9 \%)$ \\
\hline AKI, acute kidney injury; CCOT, Critical Care and Outreach.
\end{tabular}

acutely. This compares to a published mortality of $3 \%$ for non-ST elevation myocardial infarction, ${ }^{14} 7 \%$ for ST elevation myocardial infarction ${ }^{15}$ and $12 \%$ for acute stroke. ${ }^{16}$ In the setting of AKI high and low venous bicarbonates predicted a markedly worse outcome. The group with low serum bicarbonate $(<22 \mathrm{mmol} / \mathrm{L})$ had higher mortality and was more likely to be admitted to ITU or need RRT acutely than the group with bicarbonate within the normal range. Similarly those with high serum bicarbonate $(>29 \mathrm{mmol} / \mathrm{L})$ also had a near doubling of mortality and higher rates of admission to ITU but were not more likely to require acute RRT. This is perhaps not surprising as high bicarbonate almost certainly represents additional organ failure in the form of acute or chronic respiratory failure and these patients are unable to compensate for metabolic acidosis secondary to AKI. Likewise, those patients with low bicarbonate are likely to represent those with more severe illness. Regardless of the cause low or high venous bicarbonate appears to be a simple and cheap predictor (addition of this test represents a cost to our hospital of approximately $£ 1000$ over an 18 -month period) of substantial mortality which may be useful in identifying and prioritising patients at risk at an earlier stage, hopefully, increasing the opportunity to improve outcomes.

Given the poor outcomes in patients developing AKI and the lack of an effective treatment of acute tubular injury, the hope would be that early identification and intervention may reduce the injury by removing toxins and effective resuscitation. In view of this, it is intriguing that within the subgroup of patients with AKI who were reviewed by CCOT, those assessed $\geq 1$ days after their AKI alert had an increase in mortality by a factor of 2.4, were 7 times more likely to require RRT acutely and had an

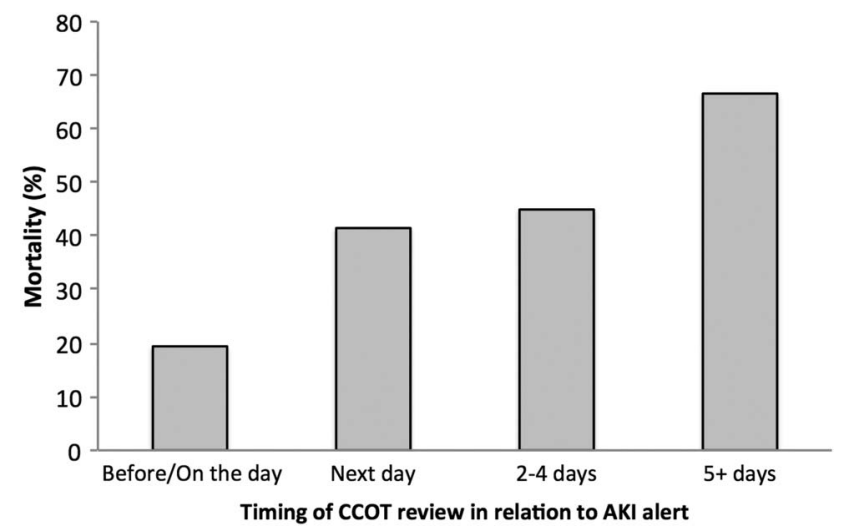

Figure 4 Increasing mortality with increasing time from acute kidney injury (AKI) alert to Critical Care and Outreach (CCOT) review. $p=0.018\left(\chi^{2}\right)$.

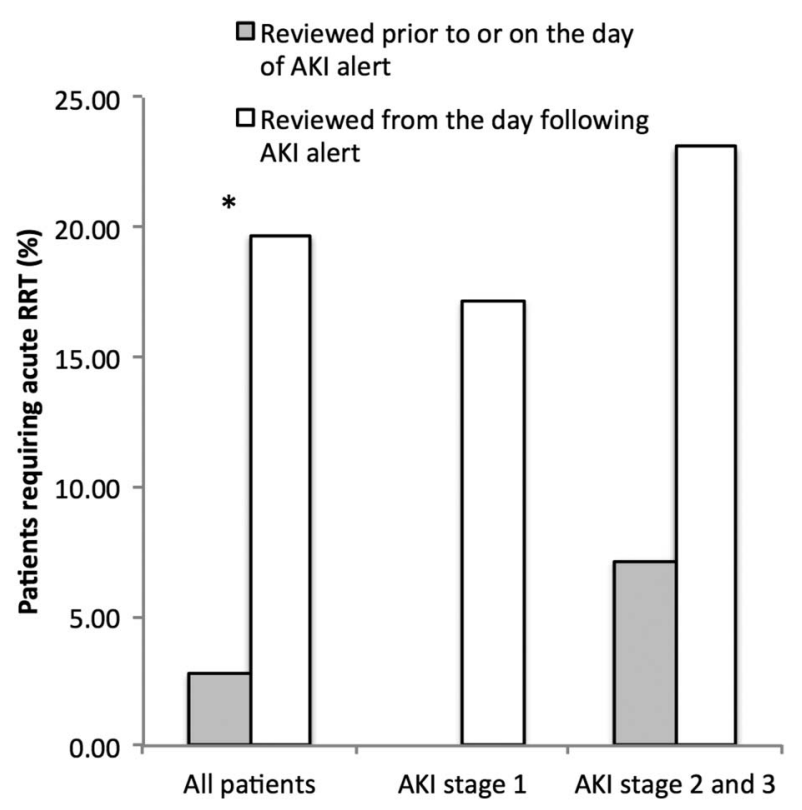

Figure 5 Increased requirement for renal replacement therapy (RRT) in patients reviewed later by Critical Care and Outreach (CCOT) following acute kidney injury (AKI) alert compared with earlier review. ${ }^{*} \mathrm{p}<0.05$.

increased length of stay by a mean of 5 days. Referral to CCOT was on the basis of physiological decline, not AKI, and it may be that those patients seen on the day of their AKI alert represent those with a more acute event prompting early and more aggressive intervention. Clearly we cannot say from a retrospective study that if patients were seen by CCOT on the day of their AKI alert, their outcomes would be better. However, given the severe consequences of AKI, adding an AKI alert to the referral indications for CCOT is very appealing, not least because for many patients CCOT referral would be nearly 2 days earlier than if left to trigger by physiological parameters.

All NHS hospitals in England must have instituted an AKI alert algorithm by March 2015 and our results offer scope for how alerts can be used either to predict those at higher risk or to improve patient outcomes. Early identification and assessment with the rapid removal of nephrotoxins and introduction of supportive measures is likely to be critical if we wish to improve outcomes in patients with AKI.

\section{Limitations}

The limitations of our study include its retrospective data collection and the likelihood of false negatives-episodes of AKI may have been missed where no creatinine was available in the last 90 days or where the creatinine was already elevated from the patient's previous baseline. The number of patients included in the subgroup reviewed by CCOT is small and there may well be confounding factors between the patients who were reviewed earlier rather than later which were not identified on retrospective analysis.

\section{Future work}

Our study shows an association between low serum bicarbonate and poorer outcomes. In order to further investigate this it would be useful to have a comprehensive multivariate analysis or to add bicarbonate to other acute illness scoring systems and identify if bicarbonate associates with poor outcomes. There was also a suggestion from our data that those patients who have progressive AKI do worse than those with AKI that do not, 
perhaps implying that the underlying pathophysiology is ongoing in the former as opposed to a single insult in the latter. Either way electronic identification of patients with progressive AKI may also help identify patients at greater risk. This could potentially be achieved by adjusting the alert algorithm to use admission creatinine as the baseline rather than the previous result directly.

\section{Main messages}

- Patients with AKI and low or high serum bicarbonate have a significantly higher mortality and are more likely to require ITU admission than those with bicarbonate within the normal range.

- A delay in review by Critical Care and Outreach is associated with significantly increased mortality, requirement for acute renal replacement therapy and length of stay.

\section{Current research questions}

- Can biochemical markers other than bicarbonate be used to risk stratify patients with AKI?

- Which supportive interventions can improve patient outcomes in AKI?

- Can a scoring system be developed to identify those at highest risk of AKI on admission to hospital?

- What are the long-term outcomes in patients who have had an episode of AKI; are there any factors that can predict those at risk of a worse outcome after discharge from hospital?

Contributors All authors have contributed substantially the body of work and fulfilled the ICMJE guidelines to grant a coauthorship. MP carried out data collection and analysis and wrote the manuscript, EB and OS-A carried out data collection, RE generated the database of AKI alerts and provided biochemical data, HM and SG made contributions to concept and design of the study, $\mathrm{MH}$ contributed to concept and design and revised the manuscript for submission.

Competing interests None declared.

Provenance and peer review Not commissioned; externally peer reviewed.

Open Access This is an Open Access article distributed in accordance with the Creative Commons Attribution Non Commercial (CC BY-NC 4.0) license, which permits others to distribute, remix, adapt, build upon this work non-commercially, and license their derivative works on different terms, provided the original work is properly cited and the use is non-commercial. See: http://creativecommons.org/ licenses/by-nc/4.0/

\section{REFERENCES}

1 Nash K, Hafeez A, Hou S. Hospital acquired renal insufficiency. Am J Kidney Dis 2002;39:930-6.

2 Liangos O, Wald R, O'Bell JW, et al. Epidemiology and outcomes of acute renal failure in hospitalized patients: a national survey. Clin J Am Soc Nephrol 2006;1:43-51.

3 Xue JL, Daniels F, Star RA, et al. Incidence and mortality of acute renal failure in Medicare beneficiaries, 1992 to 2001. J Am Soc Nephrol 2006;17:1135-42.

4 Levy EM, Viscoli CM, Horowitz RI. The effect of acute renal failure on mortality. A cohort analysis. JAMA 1996;275:1489-94.

5 Consentino F, Chaff $C$, Piedmonte M. Risk factors influencing survival in ICU acute renal failure. Nephrol Dial Transplant 1994;9:179-82.

6 Chawla LS, Amdur RL, Amodeo S, et al. The severity of acute kidney injury predicts progression to chronic kidney disease. Kidney Int 2011;79:1361-9.

7 Coca SG, Singanamala S, Parikh CR. Chronic kidney disease after acute kidney injury: a systematic review and meta-analysis. Kidney Int 2012:81:442-8.

8 Wald R, Quinn RR, Luo J. Chronic dialysis and death among survivors of acute kidney injury requiring dialysis. JAMA 2009:302:1179-85.

9 LeFrance JP, Miller DR. Acute kidney injury associates with increased long term mortality. J Am Soc Nephrol 2009;21:345-52.

10 NCEPOD report 2009. AKI: Adding insult to injury. http://www.ncepod.org.uk/ 2009aki.htm

11 Selby NM, Crowley L, Fluck RJ, et al. Use of electronic results reporting to diagnose and monitor AKI in hospitalized patients. Clin J Am Soc Nephrol 2012;7:533-40.

12 Porter CJ, Juurlink I, Bisset $\mathrm{LH}$, et al. A real time electronic alert to improve detection of acute kidney injury in a large teaching hospital. Nephrol Dial Transplant 2014;29:1888-93.

13 http://www.england.nhs.uk/wp-content/uploads/2014/06/psa-aki.pdf

14 Brieger D, Fox KA, Fitzgerald G, et al. Predicting freedom from clinical events in non-ST-elevation acute coronary syndromes: the Global Registry of Acute Coronary Events. Heart 2009;95:888.

15 Jernberg $T$, Johnson $\mathrm{P}$, Held $\mathrm{C}$, et al. Association between adoption of evidence-based treatment and survival for patients with ST-elevation myocardial infarction. JAMA 2011;305:1677-84.

16 Lee $S$, Shafe AC, Cowle MR. UK stroke incidence, mortality and cardiovascular risk management 1999-2008: time-trend analysis from the General Practice Research Database. BMJ Open 2011;1:e000269. 